\title{
Analisis Economico Del Control Biológico De La Araña Roja (Tetranychus Spp) En El Cultivo De La Rosa A Través De La Aplicación Del Hongo Entomopatogeno (Verticillium Lecanii)
}

\author{
Armando Esteban Espinoza Espinoza \\ Carla Sofía Arguello Guadalupe \\ José Luis Hidalgo Gallegos \\ Carlos Raúl Camacho Latorre
}

Docente - Investigador de la Facultad de Recursos Naturales

ESCUELA SUPERIOR POLITÉCNICA DE CHIMBORAZO, ECUADOR.

\section{Homero Santiago Robalino Robalino}

Estudiante, Facultad de Recursos Naturales

ESCUELA SUPERIOR POLITÉCNICA DE CHIMBORAZO, ECUADOR.

doi: 10.19044/esj.2017.v13n13p240 URL:http://dx.doi.org/10.19044/esj.2017.v13n13p240

\begin{abstract}
The production of roses in Ecuador is an economic opportunity that has been increasing since the 1980's. Ecuador is the fourth country in the world with a comparative advantage in rose production. Its quality and variety of colors has positioned the Ecuadorian rose as one of the best in the world. The research aims to compare four control treatments of the red spider (Tetranychus spp). Three of them are controlled through biological control (Verticillium Lecanii), while one of them is through chemical control. It tries to identify which one is the most efficient and that has the greatest economic benefit when compared to the control costs of each treatment. The results show significant evidence on T4 (chemical control), which is not only the most efficient in controlling mites at all stages of development. Also, it proves to be the least costly and it therefore provides greater profitability to the company.
\end{abstract}

Keywords: Verticillium lecanii, spider red, roses, Tetrancychus spp., biological control, economic analysis

\section{Resumen}

La producción de rosas en el Ecuador es una oportunidad económica que se ha ido incrementando a partir de los años $80 \mathrm{~s}$. Ecuador es el cuarto 
país a nivel mundial con mayor explotación de rosas, su calidad y variedad de colores ha posicionado a la rosa ecuatoriana como una de las mejores del mundo. La presente investigación busca comparar cuatro tratamientos de control de la araña roja (Tetranychus spp). Tres de ellos son a través de control biológico (Verticillium Lecanii) y una a través de control químico, buscando identificar cuál de ellos es el más eficiente y además muestra el mayor beneficio económico al compararlo con los costos de control de cada tratamiento. Los resultados muestran una evidencia significativa sobre el T4 (control químico), quien no solo es el más eficiente en el control del ácaro en todas sus etapas de desarrollo, sino que además resulta ser el menos costoso y por ende proporciona mayor rentabilidad a la empresa.

Palabras Claves: Verticillium lecanii, araña roja, control biológico, rosas, Tetranychus spp, análisis económico

\section{Introducción}

Las flores ecuatorianas son uno de los cultivos más demandados a nivel mundial, Ecuador descubrió su potencial para cultivar y exportar flores; claveles, crisantemos, gypsofilas, rosas, fueron las primeras flores que se sembraban para exportación, y ahora posee una porción importante del mercado internacional. Ecuador al ser considerado un país mega diverso por sus características biogeográfica resulta muy favorable para el desarrollo de especies como: Astromelias, claveles, crisantemos, margaritas, limonios, gipsófilas, flores de verano, etc. Las especies tropicales como la heliconia se cultivan en las áreas cálidas y húmedas del Ecuador. Las variedades de claveles que se cultivan incluyen las "Norla Barlo", "Charmeur", "Dark Organe Telster” y "Dallas”. Y una de sus especies más cotizadas las rosas se cultivan en las provincias de Pichincha, Cotopaxi, Imbabura, Cañar entre otras (Proecuador, 2013: 5).

Las rosas se convierten en el cultivo líder en la exportación de flores, con más de 60 variedades entre ellas rosa roja "First Red", "Classi", "Dallas", "Mahalia”, "Madame Del Bar" y "Royal Velvet”. Las variedades de la rosa amarilla que se cultivan son la "Allsmer Gold" y la "Skyline". Otras incluyen la rosa de pimpollos "Anna Nubia” y las de color púrpura "Ravel” y "Gretta". Los colores y tamaños son muy variados y eso ha permitido que este cultivo encabece la lista de flores con mayor demanda internacional (Proecuador, 2013: 6).

Sin embrago este cultivo es muy sensible a plagas y enfermedades que ocasionan disminución en la productividad del cultivo y requieren de altos costos económicos en el momento de realizar sus control. Entre las plagas que afecta a este cultivo se puede mencionar a pulgones o afidos, Trips, nematodos y la araña roja. De igual manera este cultivo se ve afectado 
por enfermedades como: Mildiu velloso o tizón, Oídio, Roya, Moho gris, Agallas o tumores, Mosaicos foliares entre otros (Moorman, 2016: 1)

Esta investigación se focaliza en la plaga de la araña roja (Tetranychus spp), pertenece al orden Achari, familia Tetranychidae, los tarsos terminan generalmente en uñas y empodios acompañados por setas que son de valor taxonómico. Además influyen taxonómicamente el número y posición de las setas en las patas (RICAURTE, 2002: 63). Durante su ciclo de vida atraviesa por cuatro estados de desarrollo antes de llegar a su adultez (huevo, un instar larval y dos estadios ninfales) (Robalino, 2001: 18).

Estos ácaros tienen el aparato bucal modificado para extraer altos volúmenes de jugos celulares. Esto produce manchas amarillas o blancas diminutas sobre la superficie de las hojas. Cuando comienzan a aparecer muchas de estas manchas, el follaje toma un color amarillo o bronceado, posteriormente las hojas se desprenden prematuramente. El daño es importante en los primeros estados de desarrollo de la planta, puede ocasionar un retraso en su crecimiento, disminución de la producción y calidad de la misma. En casos extremos de grandes poblaciones de araña roja llegan a secar la plata por completo (Shetlar, 2000: 8).

La Araña roja Tetranychus spp es una plaga a nivel nacional que afecta a cultivos hortícolas, frutales y florícolas, entre los más representativos. Es una plaga muy difícil de controlar pues su caparazón está compuesto por quitina, la cual le sirve como protección frente a los productos aplicados, impidiendo que el químico penetre en el ácaro y pueda matarlo. Además la “araña roja” como es conocido, adquiere rápidamente resistencia a los diferentes productos utilizados para su control, por lo que las soluciones químicas evaluadas resultan ser no duraderas, costosas, y amenazan a la productividad de los suelos (Castro, 2010: 19).

En los diferentes cultivos comerciales los ácaros ocasionan anualmente pérdidas económicas del 18 al 20\%. En Ecuador con el afán de controlar esta plaga, en rosas se invierten 20 mil dólares mensualmente y se han venido aplicando a través de los años productos químicos como floramite, hexmite, dicarsol, sinosine, kanemite entre otros (INIAP, 2005: 1), los cuales son nocivos para el suelo porque lo deterioran, tornándolo no apto para la producción; además contaminan las aguas subterráneas por infiltración, el aire y el entorno en general (Martinez \& Otros, 2006: 99).

Por esto el camino apropiado para la naturaleza es realizar un control biológico, mismo que puede ser a través de enemigos naturales o productos creados en el laboratorio con el fin de atacar a plagas con alta resistencia. Estos productos deben considerar la gran resistencia de la misma, su gran adaptabilidad a temperaturas entre $5^{\circ} \mathrm{C}$ a $42{ }^{\circ} \mathrm{C}$, el incremento de la población en alta humedad relativa y a la vez a la adaptabilidad de la plaga a baja humedad relativa (Robalino, 2004: 11). 
El hongo Entomopatógeno Verticillium lecanii, fue desarrollado con este propósito, se caracteriza por la formación de micelio septado con producción de conidias, de aproximadamente 0,5 a 0,8 micras de diámetro o mediante reproducción asexual, en conidióforos que nacen a partir de hifas ramificadas (Laverlam, 2000: 13). El hongo se obtiene por métodos artesanales sobre soporte sólido y en medios líquidos, los cuales se preparan a partir de desechos y subproductos de la industria azucarera y otros subproductos agrícolas (Fernández, 1997: 25).

\section{Metodología}

Se trabajó en un cultivo establecido comercial de rosa de la variedad Leonidas, de seis años de edad aproximadamente. El agente de control biológico fue el hongo Verticillium lecanii, de la casa Laverlam, en dosis de 0,19; 0,25 y 0,31 g por litro de agua, con un volumen de 10 litros de mezcla por cama y 250 - 300 psi de presión. Las aspersiones se hicieron con una bomba a motor de 3HP conectado con 2 lanzas de cuatro pares de boquillas Albuz ATR.

Para identificar la efectividad del hongo Verticillium lecanii en el control de este ácaro, se realizó una estimación de la población de la araña roja en el cultivo antes de la aplicación del hongo, para ello se tomó un tercio inferior, medio y superior de ocho sitios distribuidos uniformemente en la cama, se realizó una distribución en 24 hojas muestreadas en la cama, obteniendo el número de huevos y móviles por hoja. Conocido este dato se procedió a realizar las aplicaciones en las dosis indicadas y a través del monitoreo y contabilización de huevos y formas móviles se identificó el nivel de efectividad y daño a la plaga.

Se realizó una comparación de la eficiencia de los tratamientos biológicos con el control químico convencional, se hicieron tres aplicaciones químicas para mantener los niveles poblacionales de la plaga y los niveles de daño bajos. Se utilizaron rotaciones de acaricidas químicos de diferente mecanismo de acción. Para determinar la eficiencia se utilizó la fórmula propuesta por Henderson y Tilton.

$\%$ Eficacia $=100 \llbracket 1-\frac{T d * C a}{C d * T a} \rrbracket$, donde

Td: Individuos vivos después del tratamiento

Ta: Individuos vivos antes del tratamiento

Ca: Individuos vivos en el testigo absoluto antes del tratamiento

Cd: Individuos vivos en el testigo absoluto después del tratamiento

Para el análisis económico se calcularon los costos directos e indirectos por aplicación para cada uno de los tratamientos biológicos y químicos. Centralizando el número de aplicaciones durante el ensayo, para posteriormente identificar el análisis costo- beneficio e identificar los 
ingresos netos por cada tratamiento y así identificar que tratamiento proporciona mayor rentabilidad para la empresa.

\section{Resultados}

Se realizaron tres tratamientos biológicos, con las diferentes dosificaciones de aplicación del hongo benéfico. Adicionalmente se hizo un tratamiento utilizando el control químico convencional de la finca. El testigo absoluto fue utilizado exclusivamente para la determinación de la eficiencia de los acaricidas químicos y del factor de multiplicación de la plaga. Los tratamientos propuestos fueron los siguientes:

Cuadro 1. Tratamientos utilizados en la investigación

\begin{tabular}{|c|c|c|c|}
\hline TRATAMIENTO & $\begin{array}{c}\text { AGENTE } \\
\text { REGULADOR }\end{array}$ & DOSIS & FRECUENCIA \\
\hline T1 & Verticillium lecanii & $0,19 \mathrm{~g} /$ litros de agua & Semanal \\
\hline T2 & Verticillium lecanii & $0,25 \mathrm{~g} /$ litros de agua & Semanal \\
\hline T3 & Verticillium lecanii & $0,31 \mathrm{~g} /$ litros de agua & Segúnal monitoreo \\
\hline T4 & Testigo químico & $\begin{array}{c}\text { Dependiendo del } \\
\text { producto }\end{array}$ & Según \\
\hline
\end{tabular}

Realizado por: Robalino Robalino Homero Santiago

Cuadro 2. Características de los acaricidas químicos usados en el T4

\begin{tabular}{|c|c|c|c|c|}
\hline $\begin{array}{c}\text { Nombre } \\
\text { comercial }\end{array}$ & Principio Activo & Tipo & Modo de acción & Dosis utilizada \\
\hline Catombe & Abamectina & Adulticida & $\begin{array}{c}\text { Contacto, } \\
\text { translaminar }\end{array}$ & $0,5 \mathrm{cc} / \mathrm{lt}$ \\
\hline Atrim & $\begin{array}{c}\text { Lambda } \\
\text { cihalarotrina }\end{array}$ & Adulticida & Contacto, ingestión & $0,6 \mathrm{cc} / \mathrm{lt}$ \\
\hline Mavrik & Tau fluvalinato & Adulticida & Contacto, ingestión & $0,3 \mathrm{cc} / \mathrm{lt}$ \\
\hline
\end{tabular}

Realizado por: Robalino Robalino Homero Santiago

Para la verificación de la eficiencia del Hongo, se contabilizó la población de Araña Roja al iniciar cada uno de los tratamientos, realizando un análisis de varianza, con el objetivo de determinar si existe o no diferencias en el número de huevos y móviles entre tratamiento, estos resultados se muestran en el Cuadro 3

Cuadro 3: Huevos y móviles por hoja presentes previo al inicio de la investigación

\begin{tabular}{|c|c|c|c|c|c|}
\hline $\begin{array}{c}\text { Unidad } \\
\text { experimental }\end{array}$ & $\begin{array}{c}\text { Media } \\
\text { Huevos/ } \\
\text { hoja }\end{array}$ & $\begin{array}{c}\text { Media } \\
\text { Móviles/hoja }\end{array}$ & $\begin{array}{c}\text { Media } \\
\text { Huevos/hojas }\end{array}$ & $\begin{array}{c}\text { Media } \\
\text { Móviles/hoja }\end{array}$ & $\begin{array}{c}\text { Nivel } \\
\text { de } \\
\text { Daño }\end{array}$ \\
\hline T1 R1 & 1,88 & 1,75 & & & \\
\hline T1 R2 & 1,83 & 1,42 & 1,78 & 1,64 & B \\
\hline T1 R3 & 1,63 & 1,75 & & & \\
\hline T2 R1 & 1,71 & 1,38 & & & \\
\hline T2 R2 & 2,08 & 2,04 & 2,01 & 1,74 & B \\
\hline T2 R3 & 2,25 & 1,79 & & & \\
\hline T3 R1 & 2,42 & 2,13 & & & \\
\hline T3 R2 & 1,75 & 1,46 & 1,92 & 1,82 & B \\
\hline T3 R3 & 1,58 & 1,88 & & & \\
\hline T4 R1 & 2,17 & 1,96 & 1,99 & 1,88 & B \\
\hline
\end{tabular}




\begin{tabular}{|l|l|l|l|l|l|}
\hline T4 R2 & 1,88 & 1,54 & & & \\
T4 R3 & 1,92 & 2,13 & & & \\
\hline
\end{tabular}

Realizado por: Robalino Robalino Homero Santiago

En el análisis de la varianza no se encontró diferencias estadísticamente significativas entre las poblaciones iniciales de huevos y móviles por hoja tanto entre tratamientos como repeticiones. Este procesamiento es importante ya que se garantiza el inicio de la investigación con poblaciones homogéneas de la plaga asegurando resultados más fiables, esto se observa en el cuadro 4 y 5

Cuadro 4. Análisis de varianza para las poblaciones iniciales de huevos por hojas en los tratamientos biológicos y químicos

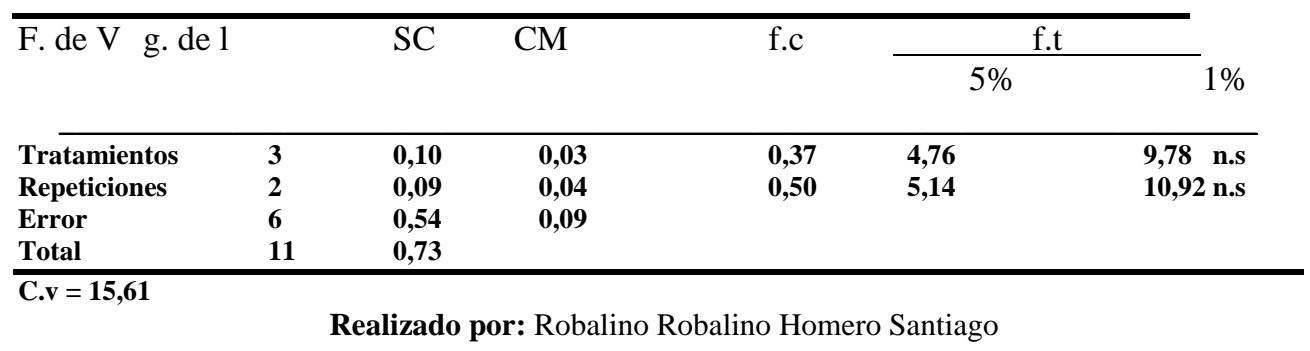

Cuadro 5. Análisis de varianza para las poblaciones iniciales de móviles por hojas en los tratamientos biológicos y químicos

\begin{tabular}{|c|c|c|c|c|c|c|}
\hline \multirow[t]{2}{*}{ F. de $\mathrm{V}$ g. de $\mathrm{l}$} & & \multirow[t]{2}{*}{$\mathrm{SC}$} & \multirow[t]{2}{*}{$\mathrm{CM}$} & \multirow[t]{2}{*}{ f.c } & \multicolumn{2}{|c|}{ f.t } \\
\hline & & & & & $5 \%$ & $1 \%$ \\
\hline Tratamientos & 3 & 0,10 & 0,03 & 0,35 & 4,76 & 9,78 n.s \\
\hline Repeticiones & 2 & 0,16 & 0,08 & 0,85 & 5,14 & 10,92 n.s \\
\hline Error & 6 & 0,55 & 0,09 & & & \\
\hline Total & 11 & 0,81 & & & & \\
\hline
\end{tabular}

C.v $=17,15$

Realizado por: Robalino Robalino Homero Santiago

Los ácaros atacados por $V$. lecanii, inicialmente se paralizan sin presentar señales externas de parasitación. Luego de aproximadamente tres a cinco días, según las condiciones ambientales, se observa que el cuerpo del arácnido se cubre de un micelio blanco grisáceo de crecimiento abundante. Es ahí cuando se los centraliza como ácaros parasitados.

Durante el desarrollo de la presente investigación, no se detectó en ningún momento parasitación del hongo sobre huevos y larvas. Los instares más susceptibles a la parasitación fueron los dos estados: Ninfales y el adulto. Esto observamos en el siguiente cuadro. 
Cuadro 6. Numero de móviles parasitados por hoja por Verticillium lecanii

\begin{tabular}{|c|c|c|}
\hline Unidad experimental & $\begin{array}{c}\text { Media de móviles } \\
\text { parasitados por unidad } \\
\text { experimental }\end{array}$ & $\begin{array}{c}\text { Media de móviles } \\
\text { parasitados por tratamiento }\end{array}$ \\
\hline T1 R1 & 0,04 & \multirow{2}{*}{0,09} \\
\hline T1 R2 & 0,15 & \multirow{2}{*}{0,13} \\
\hline T1 R3 & 0,07 & \multirow{2}{*}{0,16} \\
\hline T2 R1 & 0,10 & \\
\hline T2 R2 & 0,12 & \\
\hline T2 R3 & 0,17 & \\
\hline T3 R1 & 0,16 & \\
\hline T3 R2 & 0,17 & \\
\hline T3 R3 & 0,15 & \\
\hline
\end{tabular}

Realizado por: Robalino Robalino Homero Santiago

Pese a que la parasitación parece baja, comprendida entre los 0,09 ácaros/hoja para T1, 013 ácaros/ hojas para T2 y 0,16 ácaros/hoja para T3, los niveles de control se mantuvieron satisfactoriamente, en especial cuando la humedad relativa se mantuvo sobre 70\%. Es importante recalcar que T3 alcanzó la mayor parasitación durante las tres últimas semanas, en las que se registraron condiciones ambientales adversas para el desarrollo del hongo, es decir el control fue más perdurable.

Cuadro 7. Análisis de varianza para los móviles parasitados por hoja por tratamiento biológico

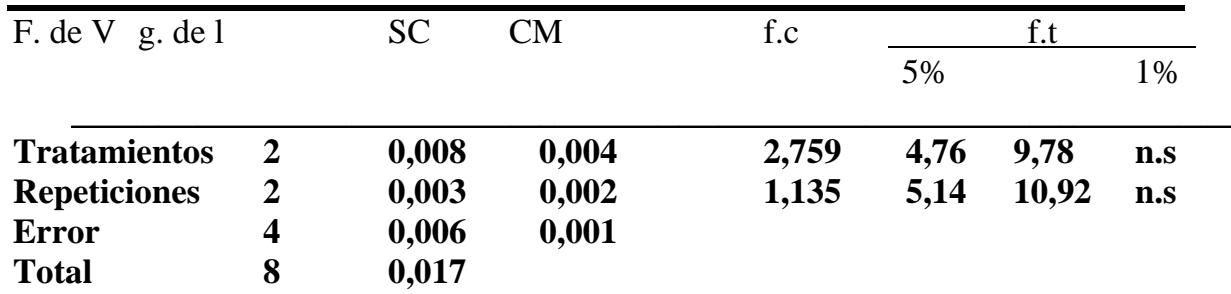

C.v $=24,33$

Realizado por: Robalino Robalino Homero Santiago

Como se puede observar no hay diferencia estadísticamente significativas en la parasitación entre los tratamientos biológicos correspondientes a las tres dosis aplicadas del hongo, lo cual habla de niveles parecidos de parasitación aun con dosis bajas.

También se logró determinar la población de huevos y móviles por hoja y los niveles de daño durante el ensayo, en todos los tratamientos el número de huevos fue similar a la cantidad de móviles por hoja, pese a que en todos los tratamientos el nivel de afectación en función a la cantidad de móviles hallados por hoja fue bajo, no se puede negar daño en algunos de los tratamientos. Esto se analiza en el siguiente cuadro: 
Cuatro 8. Numero de huevos y móviles promedio por hoja registrados durante el ensayo

\begin{tabular}{|c|c|c|c|c|c|c|}
\hline $\begin{array}{c}\text { Unidad } \\
\text { Experimental }\end{array}$ & $\begin{array}{c}\text { Media } \\
\text { huevos/hoja }\end{array}$ & $\begin{array}{c}\text { Media } \\
\text { huevos/tratamiento }\end{array}$ & $\begin{array}{c}\text { Media } \\
\text { móviles/hoja }\end{array}$ & $\begin{array}{c}\text { Nivel } \\
\text { de } \\
\text { daño }\end{array}$ & $\begin{array}{c}\text { Media } \\
\text { móviles/tratamiento }\end{array}$ & $\begin{array}{c}\text { Nivel } \\
\text { de } \\
\text { daño }\end{array}$ \\
\hline T1R1 & 7,25 & \multirow{3}{*}{8,89} & 8,28 & Bajo & \multirow{3}{*}{8,87} & Bajo \\
\hline T1R2 & 9,14 & & 9,18 & Bajo & & Bajo \\
\hline T1R3 & 10,27 & & 9,15 & Bajo & & Bajo \\
\hline T2R1 & 6,31 & \multirow{3}{*}{8,45} & 5,49 & Bajo & \multirow{3}{*}{7,37} & Bajo \\
\hline T2R2 & 8,60 & & 7,36 & Bajo & & Bajo \\
\hline T2R3 & 10,44 & & 9,25 & Bajo & & Bajo \\
\hline T3R1 & 8,99 & \multirow{3}{*}{6,78} & 9,08 & Bajo & \multirow{3}{*}{6,45} & Bajo \\
\hline T3R2 & 5,65 & & 5,20 & Bajo & & Bajo \\
\hline T3R3 & 5,69 & & 5,06 & Bajo & & Bajo \\
\hline T4R1 & 2,76 & \multirow{3}{*}{2,90} & 2,53 & Bajo & \multirow{3}{*}{2,38} & Bajo \\
\hline T4R2 & 2,93 & & 2,08 & Bajo & & Bajo \\
\hline T4R3 & 3,02 & & 2,54 & Bajo & & Bajo \\
\hline
\end{tabular}

Realizado por: Robalino Robalino Homero Santiago

Al realizar la comparación propuesta en el T4 (testigo químico) Vs T1, T2, T3 (testigos biológicos), en la población de huevos, resulta altamente significativa, por lo que se puede decir que el control químico de huevos es notablemente superior al control biológico, esto se debe a que en ningún instante se halló parasitación sobre oviposturas y por ello la población de huevos creció sin control, por lo que presentaron cantidades muy altas de ellos, contrario a lo que paso con el testigo químico.

Cuadro 9. Comparación ortogonal para las poblaciones de huevos

\begin{tabular}{lllllllc}
\hline F. de V g. de l & & SC & CM & f.c & \multicolumn{2}{c}{ f.t } & \\
\cline { 6 - 8 } & & & & & & & \\
& & & & & & & \\
Tratamientos & 3 & $\mathbf{7 4 , 2 2}$ & $\mathbf{2 4 , 7 4}$ & $\mathbf{8 , 1 1}$ & $\mathbf{4 , 7 6}$ & $\mathbf{9 , 7 8}$ & $*$ \\
T4 vs T1T2T3 & $\mathbf{1}$ & $\mathbf{6 6 , 8 0}$ & $\mathbf{6 6 , 8 0}$ & $\mathbf{2 1 . 8 9}$ & $\mathbf{5 , 9 9}$ & $\mathbf{1 3 , 7 5}$ & $*$ \\
T1 vs T2T3 & $\mathbf{1}$ & $\mathbf{3 , 2 3}$ & $\mathbf{3 , 2 3}$ & $\mathbf{1 , 0 6}$ & & & n.s \\
T2 vs T3 & $\mathbf{1}$ & $\mathbf{4 , 1 8}$ & $\mathbf{4 , 1 8}$ & $\mathbf{1 , 3 7}$ & & & n.s \\
Error & $\mathbf{6}$ & $\mathbf{1 8 , 3 1}$ & $\mathbf{3 , 0 5}$ & & & & \\
\hline
\end{tabular}

Realizado por: Robalino Robalino Homero Santiago

Por otro lado, la comparación en el T4 (testigo químico) Vs T1, T2, T3 (testigos biológicos), en la población de móviles resulta altamente significativa, por lo que se puede decir que el control de químico de forma móviles de ácaros es más eficiente que el biológico, esto debido a que durante el desarrollo del ensayo las condiciones ambientales fueron desfavorables para la acción del agente de control biológico. 
Cuadro 10. Comparación ortogonal para las poblaciones de móviles

\begin{tabular}{|c|c|c|c|c|c|c|c|}
\hline \multicolumn{2}{|c|}{ F. de V g. de $\mathrm{l}$} & \multirow[t]{2}{*}{ SC } & \multirow[t]{2}{*}{$\mathrm{CM}$} & \multirow[t]{2}{*}{ f.c } & \multicolumn{3}{|c|}{ f.t } \\
\hline & & & & & \multicolumn{2}{|c|}{$5 \%$} & $1 \%$ \\
\hline Tratamientos & 3 & 69,40 & 23,13 & 7,93 & 4,76 & 9,78 & * \\
\hline T4 vs T1T2T3 & 1 & 60,42 & 60,42 & 20,71 & 5,99 & 13,75 & $* *$ \\
\hline T1 vs T2T3 & 1 & 7,70 & 7,70 & 2,64 & & & n.s \\
\hline T2 vs T3 & 1 & 1,28 & 1,28 & 0,44 & & & n.s \\
\hline Error & 6 & 17,51 & 2,92 & & & & \\
\hline
\end{tabular}

Realizado por: Robalino Robalino Homero Santiago

Para el cálculo de la eficacia del tratamiento utilizando el testigo químico aplicado en la investigación se usó la fórmula de Henderson y Tilton, tanto para el análisis del control de huevos como de móviles esto se observa a continuación:

Cuadro 11. Porcentaje de eficacia sobre huevos de los acaricidas químicos

\begin{tabular}{|c|c|c|c|c|c|c|c|}
\hline Producto & $\begin{array}{c}\text { Td } \\
\text { (huevos/hoja) }\end{array}$ & $\begin{array}{c}\text { Ta } \\
\text { (huevos/hoja) }\end{array}$ & $\begin{array}{c}\text { Cd } \\
\text { (huevos/hoja) }\end{array}$ & $\begin{array}{c}\text { Ca } \\
\text { (huevos/hoja) }\end{array}$ & $\begin{array}{c}\text { Td * } \\
\text { Ca }\end{array}$ & $\begin{array}{c}\text { Cd* } \\
\text { Ta }\end{array}$ & $\begin{array}{c}\text { Eficacia } \\
\%\end{array}$ \\
\hline Catombe & 1,92 & 1,99 & 2,63 & 1,96 & 3,76 & 5,23 & 28,10 \\
\hline Atrim & 1,38 & 1,92 & 3,69 & 2,63 & 3,63 & 7,08 & 48,77 \\
\hline Mavrik & 2,15 & 4,60 & 17,06 & 12,25 & 26,34 & 78,48 & 66,44 \\
\hline
\end{tabular}

Realizado por: Robalino Robalino Homero Santiago

Cuadro 12. Porcentaje de eficacia sobre móviles de los acaricidas químicos

\begin{tabular}{|c|c|c|c|c|c|c|c|}
\hline Producto & $\begin{array}{c}\text { Td } \\
\text { (huevos/hoj } \\
\text { a) }\end{array}$ & $\begin{array}{c}\text { Ta } \\
\text { (huevos/hoja) }\end{array}$ & $\begin{array}{c}\text { Cd } \\
\text { (huevos/hoja) }\end{array}$ & $\begin{array}{c}\text { Ca } \\
\text { (huevos/hoja) }\end{array}$ & $\begin{array}{c}\text { Td } \\
* \\
\text { Ca }\end{array}$ & $\begin{array}{c}\text { Cd* } \\
\text { Ta }\end{array}$ & $\begin{array}{c}\text { Eficacia } \\
\%\end{array}$ \\
\hline Catombe & 2,60 & 1,88 & 2,31 & 1,42 & 3,69 & 4,34 & 14,99 \\
\hline Atrim & 1,40 & 2,60 & 4,25 & 2,31 & 3,23 & 11,05 & 70,73 \\
\hline Mavrik & 0,63 & 3,60 & 18,19 & 10,56 & 6,65 & 65,48 & 89,84 \\
\hline
\end{tabular}

Realizado por: Robalino Robalino Homero Santiago

Para la presente investigación es importante analizar el índice tallos/plantas/semana, este es el indicador de productividad más utilizado en el cultivo ornamental. En el caso particular de la investigación se analizó el índice tallo/planta/semana/tratamiento, el cual se obtuvo dividiendo la producción semanal por tratamiento para el número de plantas del mismo, que es de 720. En general el manejo de la finca se fundamenta en la producción abierta por lo que los índices semanales de producción no varían mucho y no se muestran picos de cosecha en fechas específicas, por ello el análisis de producción se lo hizo semanal. 
Cuadro 13. Producción semanal e índice tallos/planta/semana/tratamiento

\begin{tabular}{|c|c|c|c|c|c|c|c|c|}
\hline \multirow{2}{*}{ Semana } & \multicolumn{3}{|c|}{ Tallos cosechados por semana } & \multicolumn{4}{c|}{ Índice tallos/planta/semana } \\
\cline { 2 - 9 } & $\mathrm{T} 1$ & $\mathrm{~T} 2$ & $\mathrm{~T} 3$ & $\mathrm{~T} 4$ & $\mathrm{~T} 1$ & $\mathrm{~T} 2$ & $\mathrm{~T} 3$ & $\mathrm{~T} 4$ \\
\hline 8 & 78 & 71 & 82 & 78 & 0,11 & 0,10 & 0,11 & 0,11 \\
\hline 9 & 76 & 88 & 94 & 90 & 0,11 & 0,12 & 0,13 & 0,13 \\
\hline 10 & 82 & 88 & 89 & 84 & 0,11 & 0,12 & 0,12 & 0,12 \\
\hline 11 & 82 & 57 & 77 & 77 & 0,11 & 0,08 & 0,11 & 0,11 \\
\hline 12 & 62 & 38 & 63 & 58 & 0,09 & 0,05 & 0,09 & 0,08 \\
\hline 13 & 61 & 56 & 72 & 66 & 0,08 & 0,08 & 0,10 & 0,09 \\
\hline 14 & 56 & 55 & 80 & 75 & 0,08 & 0,08 & 0,11 & 0,10 \\
\hline 15 & 65 & 84 & 87 & 101 & 0,09 & 0,12 & 0,12 & 0,14 \\
\hline 16 & 57 & 71 & 76 & 96 & 0,08 & 0,10 & 0,11 & 0,13 \\
\hline 17 & 54 & 92 & 71 & 105 & 0,08 & 0,13 & 0,10 & 0,15 \\
\hline 18 & 48 & 61 & 64 & 82 & 0,07 & 0,09 & 0,09 & 0,11 \\
\hline Media & 65,57 & 69,30 & 77,73 & 82,93 & 0,09 & 0,10 & 0,11 & 0,12 \\
\hline
\end{tabular}

Realizado por: Robalino Robalino Homero Santiago

El daño en tallos fue mínimo lo que es fundamental al momento de escoger si el cultivo se venderá en el mercado nacional o internacional, durante el análisis de la semana (8 a la 18) los resultados fueron: En el caso del T1: 24 individuos, con una media de 2,18 en el período analizado, T2: 21 individuos con una media de 1,91, T3: 18 individuos con una media de 1,64, y T4: 11 individuos con una media de 1.

Para el análisis económico se consideró la producción mantenida a lo largo de once semanas que duro la investigación, recordando que no se trata de una producción sincronizada (pisos), sino más bien abierta. El siguiente cuadro recoge los valores medios de productividad semanal y flor para mercado nacional e internacional por daño de ácaros por tratamiento.

Cuadro 14. Producción exportable y proyección de producción a una hectárea por tratamiento

\begin{tabular}{|c|c|c|c|c|c|}
\hline $\begin{array}{c}\text { Tratamien } \\
\text { to }\end{array}$ & $\begin{array}{c}\text { Media tallos } \\
\text { cosechados/sem } \\
\text { ana }\end{array}$ & $\begin{array}{c}\text { Media flor } \\
\text { nacional/sem } \\
\text { ana }\end{array}$ & $\begin{array}{c}\text { Media } \\
\text { semanal } \\
\text { exportab } \\
\text { le }\end{array}$ & $\begin{array}{c}\text { Total tallos } \\
\text { exportables/ci } \\
\text { clo }\end{array}$ & $\begin{array}{c}\text { Total tallos } \\
\text { exportables/ci } \\
\text { clo }\end{array}$ \\
\hline T1 & 65,57 & 2,18 & 63,39 & 697,34 & 62748,98 \\
\hline T2 & 69,30 & 1,91 & 67,39 & 741,32 & 66706,74 \\
\hline T3 & 77,73 & 1,64 & 76,09 & 836,96 & 75312,45 \\
\hline T4 & 82,93 & 1,00 & 81,93 & 901,27 & 81099,58 \\
\hline
\end{tabular}

Realizado por: Robalino Robalino Homero Santiago

Para realizar el análisis económico se identificó los costos de mano de obra y los costos de productos aplicados en los cuatro tratamientos. Identificando que los costos de mano de obra de tratamientos biológicos son más altos debido al número de repeticiones, las cuales fueron en total 10 aplicaciones por cada uno de los tratamientos de acuerdo a la dosificación antes mencionada. En el caso de los costos de los productos aplicados se realizó un análisis de los tres productos químicos utilizados en las dosis 
recomendadas en un total de tres aplicaciones y se identificó una clara diferencia que se demuestra a continuación:

Cuadro 15. Costo tratamiento biológico

\begin{tabular}{|c|c|c|c|c|c|c|c|c|}
\hline Tratamiento & $\begin{array}{l}\text { Agente } \\
\text { Regulador }\end{array}$ & $\begin{array}{c}\text { Dosis } \\
\text { utilizada }\end{array}$ & $\begin{array}{c}\text { Costo } \\
\text { producto }\end{array}$ & Costo/gr & $\begin{array}{c}\text { Costo } \\
\text { tratamiento }\end{array}$ & $\begin{array}{r}\text { Costo total } \\
\text { (T1,T2,T3) }\end{array}$ & $\begin{array}{c}\text { Costo } \\
\text { Mano } \\
\text { de } \\
\text { Obra } \\
\end{array}$ & $\begin{array}{c}\text { Costo } \\
\text { Total/parcela }\end{array}$ \\
\hline $\mathrm{T} 1$ & $\begin{array}{c}\text { Verticillium } \\
\text { lecanii }\end{array}$ & $\begin{array}{c}\text { 0,19g/litros } \\
\text { de agua }\end{array}$ & 62,62 & 0,12524 & 0,0237956 & 5,710944 & & \\
\hline $\mathrm{T} 2$ & $\begin{array}{c}\text { Verticillium } \\
\text { lecanii }\end{array}$ & $\begin{array}{c}0,25 \mathrm{~g} / \text { litros } \\
\text { de agua }\end{array}$ & 62,62 & 0,12524 & 0,03131 & 7,5144 & & \\
\hline T3 & $\begin{array}{c}\text { Verticillium } \\
\text { lecanii }\end{array}$ & $\begin{array}{c}0,31 \mathrm{~g} / \text { litros } \\
\text { de agua }\end{array}$ & 62,62 & 0,12524 & 0,0388244 & 9,317856 & 200 & \\
\hline \multicolumn{6}{|c|}{ Totales } & 22,5432 & 200 & 222,5432 \\
\hline
\end{tabular}

Realizado por: Robalino Robalino Homero Santiago

El costo total de los tres tratamientos biológicos por parcela es de $\$ 222,5432$ por la parcela utilizada para el ensayo, lo que trasformado a costo/hectáreas es 4764,95. En el caso del tratamiento Químico los costos son:

Cuadro 16. Costo tratamiento químico

\begin{tabular}{|c|c|c|c|c|c|c|c|c|}
\hline $\begin{array}{l}\text { Nombre } \\
\text { comercial }\end{array}$ & $\begin{array}{c}\text { Principio } \\
\text { Activo }\end{array}$ & $\begin{array}{c}\text { Dosis } \\
\text { utilizada }\end{array}$ & $\begin{array}{c}\text { Costo } \\
\text { producto/lt }\end{array}$ & Costo/cc & $\begin{array}{c}\text { Costo } \\
\text { Tratamiento/lt }\end{array}$ & $\begin{array}{l}\text { Costo } \\
\text { Total } \\
\text { (T4) }\end{array}$ & $\begin{array}{c}\text { Costo } \\
\text { Mano } \\
\text { de } \\
\text { Obra }\end{array}$ & $\begin{array}{c}\text { Costo } \\
\text { Total/parcela }\end{array}$ \\
\hline Catombe & Abamectina & $0,5 \mathrm{cc} / \mathrm{lt}$ & $\$ 150$ & 0,15 & 0,225 & 54 & \multirow{3}{*}{60} & \\
\hline Atrim & $\begin{array}{c}\text { Lambda } \\
\text { cihalarotrina }\end{array}$ & 0,6 cc/lt & $\$ 30$ & 0,03 & 0,054 & 12,96 & & \\
\hline Mavrik & $\begin{array}{c}\text { Tau } \\
\text { fluvalinato }\end{array}$ & 0,3 cc/lt & $\$ 32$ & 0,032 & 0,0288 & 6,912 & & \\
\hline \multicolumn{6}{|c|}{ Totales } & 73,872 & 60 & 133,872 \\
\hline
\end{tabular}

Realizado por: Robalino Robalino Homero Santiago

El costo total de los T4 tratamientos químico por parcela es de \$ 133,872 por la parcela utilizada para el ensayo, lo que trasformado a costo/hectáreas es 2863,24. Los datos anteriores claramente evidencian una muestra significativa en la diferencia de costos de aplicar los tratamientos biológicos vs el tratamiento químico.

En lo que se refiere a ingresos, el precio de la rosa ecuatoriana en el mercado nacional fluctúa dependiendo de la época, entre un precio de \$0,5 centavos y \$1 dólar. Por el contrario a nivel internacional su precio varía de acuerdo al destino final, variedad, color, tamaño del tallo, entre otros. Manteniéndose Ecuador como uno de los principales países exportadores de rosa, siendo nuestro principal mercado Estados Unidos, seguido de Europa y Rusia los precios están desde \$1 a 3,96 en países como Francia, esto explica porque más del 50\% de la producción nacional de la rosa se exporta, Según datos de Proecuador solo en Febrero del 2017 se envió 13 mil toneladas de 
rosas al mundo. Los resultados de ingresos del experimento se presentan a continuación:

Cuadro 17. Ingresos Netos

\begin{tabular}{|c|c|c|c|c|c|}
\hline DENOMINACIÓN & UNIDADES & $\mathrm{T} 1$ & $\mathrm{~T} 2$ & T3 & $\mathrm{T} 4$ \\
\hline $\begin{array}{c}\text { RENDIMIENTO } \\
\text { TOTAL }\end{array}$ & Tallos/Trat./ciclo/ha & 62748,98 & 66706,74 & 75312,45 & 81099,58 \\
\hline $\begin{array}{l}\text { RENDIMIENTO } \\
\text { AJUST (15\%) }\end{array}$ & Tallos/Trat./ciclo/ha & $\begin{array}{c}53336,63 \\
3 \\
\end{array}$ & $\begin{array}{c}56700,72 \\
9\end{array}$ & $\begin{array}{c}64015,58 \\
25\end{array}$ & $\begin{array}{c}68934,64 \\
3 \\
\end{array}$ \\
\hline PRECIO DE VENTA & USA/tallo nacional & 0,75 & 0,75 & 0,75 & 0,75 \\
\hline PRECIO DE VENTA & USA/tallo exportado & 2,34 & 2,34 & 2,34 & 2,34 \\
\hline $\mathrm{BBC}$ & $\begin{array}{c}\text { USA/Ha/Tratamiento/nacio } \\
\text { nal }\end{array}$ & $\begin{array}{c}20001,23 \\
738 \\
\end{array}$ & $\begin{array}{c}17010,21 \\
87 \\
\end{array}$ & $\begin{array}{c}19204,67 \\
48 \\
\end{array}$ & $\begin{array}{c}20680,39 \\
29 \\
\end{array}$ \\
\hline $\mathrm{BBC}$ & $\begin{array}{l}\text { USA/Ha/Tratamiento/inter } \\
\text { nacional } \\
\end{array}$ & $\begin{array}{c}62403,86 \\
061 \\
\end{array}$ & $\begin{array}{c}66339,85 \\
293 \\
\end{array}$ & $\begin{array}{c}74898,23 \\
15 \\
\end{array}$ & $\begin{array}{c}80653,53 \\
23 \\
\end{array}$ \\
\hline $\mathrm{BBC}$ & Beneficio por tratamiento & $\begin{array}{l}82405,09 \\
799\end{array}$ & $\begin{array}{c}83350,07 \\
163\end{array}$ & $\begin{array}{l}94102,90 \\
63\end{array}$ & $\begin{array}{c}101333,9 \\
25\end{array}$ \\
\hline
\end{tabular}

Realizado por: Robalino Robalino Homero Santiago

Como se puede observar en el cuadro anterior el nivel de rentabilidad de la empresa florícola incrementa notablemente con el T4 (tratamiento químico), sin embargo con el tratamiento biológico T3 existe una menor brecha de ingresos, los cuales se ven disminuidos por los altos costos de mano de obra que encarecen los tratamientos de control biológico. Es importante aclara que si bien el uso de tratamiento químico en la presente investigación resulta ser el de mayor rentabilidad no asegura la sostenibilidad agrícola, pues como ya se conoce el uso de químicos afecta notablemente a la naturaleza y por ende al futuro desarrollo del cultivo. Lo cual deberá comprobarse con futuras investigaciones.

\section{Conclusion}

- $\quad$ Verticillium lecanii, bajo condiciones ambientales favorables, mostro ser eficaz agente de control biológico de Araña roja Tetranychus spp. En el cultivo comercial de rosa. La media de huevos y móviles registrados semanalmente en cada tratamiento comparados con el testigo absoluto, es significativamente inferior, lo que indica que existió un factor que limitó el crecimiento de la población de ácaros.

- Las comparaciones entre tratamientos biológicos para el control de oviposturas, no resultaron estadísticamente significativas por lo que se asume que cualquier dosis de aplicación del hongo presenta los mismos niveles de control. Por otro lado el control a través del T4 (testigo químico) fue altamente significativo en comparación con los otros tratamientos, concluyendo que el tratamiento químico resulta más eficiente que el control biológico.

- Los beneficios económicos calculados en los cuatro tratamientos muestran una diferencia importante, pues el T4 resulta más rentable, que los 
tratamientos biológicos; esto debido principalmente a los costos de mano de obra. Sin embargo, con la presente investigación no podemos decir que se han evaluado los daños ambientales de cada tratamiento por lo cual tampoco se podría asegurar que se está hablando de un desarrollo sustentable del cultivo.

\section{References:}

1. Castro, J. (2010). Evaluación Del Control Biológico Que Ejerce La Simbiosis Del Hongo Entomopatógeno (Paecilomyces Fumosoroseus) Junto Con El Organismo Quitinolítico Aislado A Partir De Cascarilla De Camarón Sobre El Ácaro (Tetranychus Urticae) Fitopatógeno De Rosas, Median. Quito: ESPE.

2. Fernandez, O. (1997). Microorganismos en el control fitosanitario en Cuba: Tecnologias de producción. La Habana: Instituto de Sanidad Vegetal.

3. Lavarlam (2000). Especificaciones tecnicas del producto Vertisol wp.

4. La Flor Del Ecuador (2013). Publicaiones de expoflores

5. Martinez et al. (2006). Approaches for sampling the two spotted spider mite on Clementines in Spain. España: Journal Economic entomologic.

6. Moorman, G. (01 de 2016). es.scribd.com. Recuperado el 27 de 03 de 2017, de es.scribd.com: https://es.scribd.com/document/333465648/Enfermedades-de-LaRosa

7. Proecuador (2013). Analisis Sectorial de Flores. Guayaquil-Quito: Proecuador.gob.ec.

8. Revista Lideres (2015). El 2015 es un año de ajustes para el Sector Floricultor Ecuatoriano. Disponible en http://www.revistalideres.ec/lideres/sector-floricultor-rusia-mercadoecuador.html. El Comercio.

9. Ricaurte, M. (2002). Evaluacion de productos biorracionales y productos quimicos sinteticos con dos frecuencias de aplicaciones para el control del acaro. Riobamba: ESPOCH.

10. Robalino (2004). Evaluacion Del Control Biologico De La Araña Roja Con Tres Dosis De Aplicacion Del Hongo Verticillium lecanii en el cultivo de la rosa. Riobamba: ESPOCH.

11. Robalino, H. (2001). Control Biologico de la Araña Roja con su enemigo natural. Riobamba: ESPOCH.

12. Shetlar, D. (2000). Spider mities and their control. Ohio State: Horticulture and Crop Science. 